Journal of
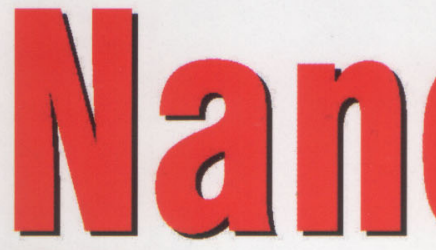

DSB
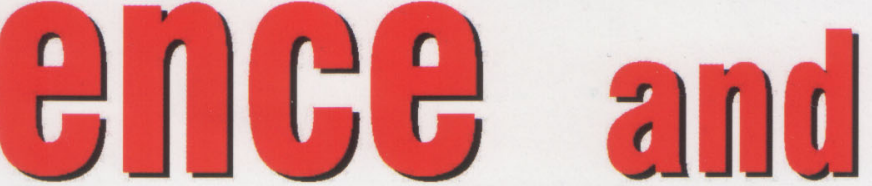

1?
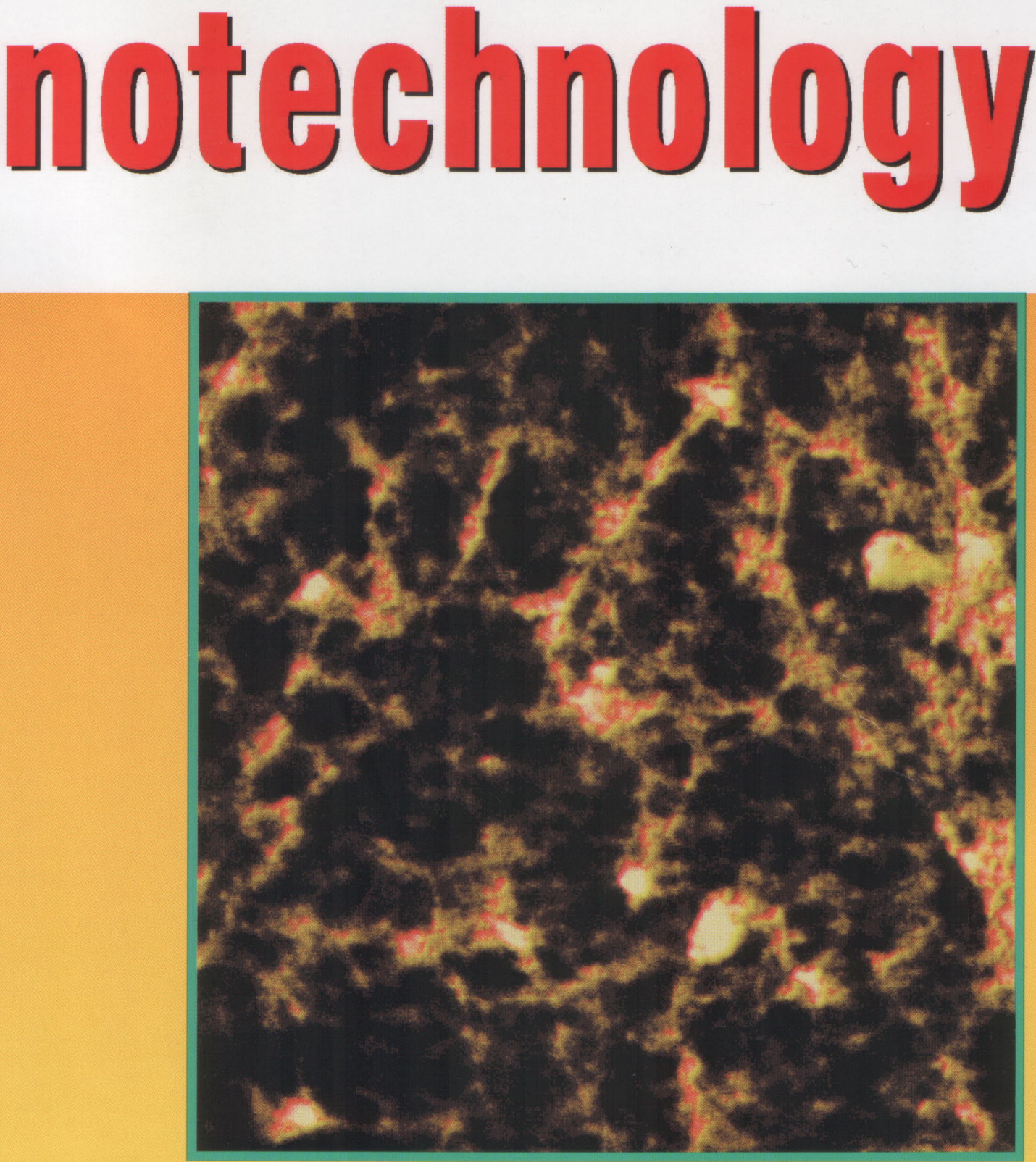

Nanoporous Aluminum Oxide for Enzyme Biosensors Self-Assembly of Tubulin with Luminescent Nanorods Manganese-doped Zinc Sulfide Nanoclusters

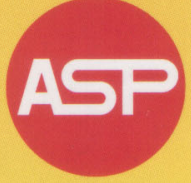
Solubilization of Carbon Nanotubes 


\title{
Visualizing the Self-Assembly of Tubulin with Luminescent Nanorods
}

\author{
Jürgen Riegler, ${ }^{\mathrm{a}}$ Peter Nick, ${ }^{\mathrm{b}}$ Udo Kielmann, ${ }^{\mathrm{a}}$ and Thomas Nann, ${ }^{\mathrm{a}, *}$ \\ ${ }^{\text {a }}$ Freiburg Materials Research Center, D-79104 Freiburg, Germany \\ ${ }^{\mathrm{b}}$ Institute for Biology II, University of Freiburg, D-79104 Freiburg, Germany
}

\begin{abstract}
We succeeded in labeling tubulin with luminescent CdSe nanorods. The labeled tubulin was still able to self-assemble into microtubules, demonstrating that the protein has remained functional. The comparison with rhodamine-labeled tubulin revealed that whereas the rhodamine bleached completely within the observed time frame, the nanorods did not show any bleaching, which makes it possible to follow the key events of self-assembly at high time resolution. Preparation and derivatization of nanorods are described. Water-soluble, protein-reactive group-containing particles were covalently coupled to tubulin. The conjugate was purified by one cycle of assembly and disassembly and used to induce the formation of fluorescent microtubules. These results suggest that the use of luminescent nanorods should allow continuous confocal monitoring of dynamic biological processes.
\end{abstract}

Keywords: Fluorescent Probes, Semiconductor Nanoparticles, Self-Assembly of Tubulin, Analytical Methods, Fluorescence Microscopy, Luminescence, Colloids.

\section{INTRODUCTION}

Because of their mesoscopic optical properties, II-VI semiconductor nanocrystals in the lower nanometer size range have recently attracted considerable attention. Because the charge carriers are confined in at least one dimension, the emission wavelength can be tuned by the size and morphology of the nanoparticles. The full width at half-maxima (FWHMs) of the photoluminescence peaks can be narrowed by increasing the monodispersity of the nanoparticles. Typically, the FWHMs range below $30 \mathrm{~nm}$. The position of the emission peaks can be tuned through the choice of the semiconductor and through the morphology and size of the nanocrystals (making use of a quantum size effect). With the use of suitable materials and morphologies the complete visible $(400-780 \mathrm{~nm})$ as well as the ultraviolet (UV) (180-400 $\mathrm{nm}$ ) and the near-infrared (NIR) (780-1200 nm) ranges can be covered by the emission of such particles. Moreover, nanoparticles broadly absorb UV light and do not bleach under excitation. These properties endow nanoparticles with an excellent potential for specific and nonspecific labeling of biological molecules (e.g., proteins) that is expected to excel that of the conventional organic fluorophores. ${ }^{1}$

In recent years some efforts have been made to exploit this potential, mainly by covalent coupling of luminescent nanocrystals to proteins-mostly antibodies. ${ }^{1-7}$ In addition, nanoparticles have been derivatized to label organelles by

"Author to whom correspondence should be addressed. nonspecific interactions. ${ }^{8,9}$ A third focus has been on the covalent coupling of oligonucleotides to luminescent nanocrystals. $^{10-15}$ These approaches were targeted mainly to optimize the coupling as well as the optical properties of the label and therefore to visualize static situations, such as the localization of proteins in fixed cells or the abundance of labeled mRNA in a given cell at the time of extraction. However, certain properties of fluorescent nanorods, for instance, the high bleaching resistance, would be manifested mainly in dynamic systems that have to be continuously followed at high resolution through the relevant time interval. Under these conditions, the bleaching of conventional fluorophores poses serious constraints to the spatial and temporal resolution of observation. Thus, the high bleaching resistance of fluorescent nanorods is expected to provide a real payoff as compared with conventional fluorophores.

To test the performance of fluorescent nanorods in the visualization of biologically relevant molecular dynamics, the self-assembly of microtubules from tubulin heterodimers was chosen as a case study. Microtubule assembly can be triggered in vitro by raising the temperature and adding GTP. It can be followed microscopically with the use of fluorescent conjugates of purified tubulin. Because the self-assembly of microtubules is a nonlinear process with a high degree of cooperativity, the time course of microtubule nucleation contains a highly stochastic component that makes it difficult to predict the exact timing of the process. To analyze the "interesting" phase of assembly (early nucleation and elongation of microtubules) there- 
fore requires continuous observation. However, when selfassembly is observed under high magnification, fluorophore bleaching usually places serious constraints on the continuous observation of the assembly process.

The present study describes how photoluminescent nanorods are synthesized and covalently coupled to tubulin, and how the self-assembly of labeled microtubules can be visualized with these nanorods. The formation of microtubules was displayed by means of fluorescence microscopy and compared with rhodamine-labeled microtubule.

\section{EXPERIMENTAL DETAILS}

\subsection{Preparation of Fluorescent Nanorods Amenable to Biological Applications}

The preparation of fluorescent nanorods that are amenable to use in biological systems requires that they be watersoluble and can be coupled to proteins. This includes four steps: (1) CdSe nanorods are synthesized. (2) They are coated with a protective, inert wurtzite shell (wurtzite is the hexagonal modification of $\mathrm{ZnS}$ ). (3) They are then further derivatized by a surrounding silica shell. (4) Their surface is coated with an additional shell that provides phosphonate and amino groups for coupling to biomolecules.

The CdSe nanorods were prepared by a recently published organometallic procedure. ${ }^{16}$ These nanorods were passivated with the following method: $100 \mathrm{mg}$ CdSe nanorods were dissolved in $4 \mathrm{~g}$ trioctylphosphineoxide (TOPO) (Avocado) and $2 \mathrm{ml}$ trioctylphosphine (TOP) (Fluka) under an inert gas atmosphere $\left(\mathrm{N}_{2}\right)$ at $90{ }^{\circ} \mathrm{C}$. When the solution was clear, the temperature was raised to $140^{\circ} \mathrm{C}$. A solution of $31 \mu \mathrm{l}$ diethyl zinc (Strem) and $61 \mu 1$ 1,1,1,3,3,3-hexamethyldisilathiane ((TMS $)_{2} \mathrm{~S}$ ) (Aldrich) in $2 \mathrm{ml}$ TOP was prepared in a drybox and transferred to a syringe. The $\mathrm{Zn}$ solution was quickly injected into the nanorod solution under Schlenk conditions (a technique that allows the handling of liquids within an inert gas atmosphere), and the mixture was stirred overnight at $90^{\circ} \mathrm{C}$. Subsequently, $5 \mathrm{ml}$ dry butanol was added, and the reaction mixture was stirred for another several hours at $60{ }^{\circ} \mathrm{C}$. The flask was allowed to cool down to room temperature, and the nanorods were precipitated with dry methanol. After centrifugation, the precipitate was washed several times with dry methanol and used directly for the subsequent steps.

The synthesis of the silica shell was based on a previously published method for gold nanoparticles ${ }^{17}$ but had to be extensively revised, because of the differences between gold nanoparticles and $\mathrm{CdSe} / \mathrm{ZnS}$ nanorods. The wurtziteprotected nanorods prepared in the previous step were dissolved in $30 \mathrm{ml}$ dry tetrahydrofuran (THF) containing $5 \mathrm{mM}$ 3-mercaptopropyltrimethoxysilane (MPS) (ABCR) and $5 \mathrm{mM}$ mercaptoethanol (Aldrich) to yield a stock stolution that was stirred overnight under Schlenk conditions. To coat the silicate shell with reactive phosphonate and amino groups, $1 \mathrm{ml}$ of this stock solution was transferred to a $10-\mathrm{ml}$ Schlenk flask and $2 \mathrm{ml}$ dry methanol was added. Successively, $17 \mu \mathrm{l}$ 3-aminopropyldimethylethoxysilane (ABCR), $67 \mu \mathrm{l}$ 3-trihydroxysilylpropylmethylphosphonate ( $42 \%$ in water, ABCR), $300 \mu \mathrm{l}$ of a methanolic solution of tetramethylamoniumhydroxyd (20\%, Fluka), and $67 \mu l$ of water were added. The reaction mixture was refluxed for $25 \mathrm{~min}$ and then cooled down to room temperature. Then $17 \mu \mathrm{l}$ trimethylchlorosilane (Aldrich) was added, and the solution was refluxed for another $10 \mathrm{~min}$. Eventually, $2 \mathrm{ml}$ of water was added, and the mixture was washed three times with $5 \mathrm{ml}$ chloroform. As final step, all small reactants were removed by extensive dialysis versus phosphate-buffered saline (PBS).

\subsection{Covalent Linkage of Nanorods to Tubulin}

Neurotubulin was prepared from fresh porcine brains by two subsequent cycles of cold-induced disassembly and warminduced reassembly following the Shelanski protocol. ${ }^{18}$ The tubulin was freed from microtubule-associated proteins (MAPs) by cation-exchange chromatography ${ }^{18}$ and checked for purity by silver stain. The MAP-free tubulin was then divided into two equal aliquots. One aliquot was conjugated to tetramethyl-rhodamine following a standard protocol. ${ }^{19}$ The other aliquot was conjugated to the amino-coated fluorescent nanorods. Five hundred microliters of the nanorod preparation was mixed with $500 \mu$ l of MAP-free tubulin (concentration adjusted to $1 \mathrm{mg} / \mathrm{ml}$ in PBS) complemented with $1 \mathrm{mM}$ phenylmethylsulfonyl fluoride (PMSF), $1 \mathrm{mM}$ dithiothreitol (DTT), and $20 \mu \mathrm{l}$ of an aqueous solution of carbodiimid $\left(50 \% \mathrm{v} / \mathrm{v}\right.$; Fluka) and incubated at $4{ }^{\circ} \mathrm{C}$ for $4 \mathrm{~h}$ in a topover shaker at $20 \mathrm{rpm}$. Denatured tubulin was precipitated by centrifugation at $15,000 \times g$ for $15 \mathrm{~min}$ in the cold $\left(4^{\circ} \mathrm{C}\right)$. The reddish supernatant was filtrated through a $0.22-\mu \mathrm{m}$ filter to remove potential tubulin aggregations and then complemented with $1 \mathrm{mM} \mathrm{GTP}, 1 \mathrm{mM} \mathrm{MgCl}{ }_{2} \cdot 6 \mathrm{H}_{2} \mathrm{O}$ and incubated at $30{ }^{\circ} \mathrm{C}$ for $30 \mathrm{~min}$ to induce the formation of sedimentable microtubules. These were collected by ultracentrifugation (10 min, $300,000 \times g, 4^{\circ} \mathrm{C}$ ). The clear and colorless supernatant was discarded, and the intensely red sediment was resuspended on ice in $100 \mu 1$ ice-cold microtubule-assembly buffer (50 mM piperazine-1,4-diethanesulfonic acid, $1 \mathrm{mM}$ $\mathrm{MgCl}_{2} \cdot 6 \mathrm{H}_{2} \mathrm{O}, 5 \mathrm{mM}$ ethyleneglycol-bis-(2-aminomethyl)tetraacetic acid, $\mathrm{pH}$ 6.8) complemented with $1 \mathrm{mM}$ PMSF and $1 \mathrm{mM}$ DTT. After incubation on ice for $15 \mathrm{~min}$, the solution was clarified by ultracentrifugation (10 min, 300,000 $\times g, 4{ }^{\circ} \mathrm{C}$ ), and the colored supernatant was frozen in liquid nitrogen until use. This supernatant thus contained tubulin that after the coupling had passed through one cycle of warminduced assembly and cold-induced disassembly.

\subsection{Induction of Microtubule Assembly}

Coverslips were coated with $0.5 \% \mathrm{v} / \mathrm{v}$ polylysine (SigmaAldrich) in a moist chamber and then warmed to $37^{\circ} \mathrm{C}$. 
MAP-free neurotubulin coupled to rhodamine or to fluorescent nanorods was thawed on ice, and the concentration adjusted with ice-cold microtubule-assembly buffer to $1 \mathrm{mg} / \mathrm{ml}$ of tubulin and passed through a $0.22-\mu \mathrm{m}$ filter to remove microtubule fragments or tubulin aggregates and kept on ice. A drop of $50 \mu \mathrm{l}$ of the tubulin solution was complemented with $1 \mathrm{mM}$ GTP, rapidly transferred to a coated, warm coverslip, and directly viewed under an epifluorescence microscope (Axioskop, Zeiss) at high magnification $(1000 \times)$. To view the nanorod-coupled tubulin, a filter set for cyano-fluorescent-protein/yellow-fluorescentprotein fluorescence resonance energy transfer (Filter set 48; Zeiss) was used with excitation, a band-pass filter (436$456 \mathrm{~nm}$ ), a beam splitter at $455 \mathrm{~nm}$, and emission through a band-pass filter $(535-565 \mathrm{~nm})$. To view the rhodaminecoupled tubulin, a conventional rhodamine filter set (Filter set 15; Zeiss) was used. The assembly of microtubules was followed under continuous excitation and recorded at intervals of $10 \mathrm{~s}$ as digital images (Axiovision; Zeiss). This time resolution was caused by the scanning time required to record the images at the high magnification and could be reduced considerably by using simultaneous rather than sequential image acquisition by a video system.

\section{RESULTS AND DISCUSSION}

\subsection{Preparation of Luminescent Nanorods}

Figure 1 shows a transmission electron micrograph (TEM) of CdSe nanorods (variations in contrast are due to different positions of the nanorods on the TEM grid). These particles were prepared at $240{ }^{\circ} \mathrm{C}$ within a growth time of $5 \mathrm{~min}$. The average aspect ratio of these nanorods was 3.5 ; the typical length was found to be between 10 and $20 \mathrm{~nm}$,

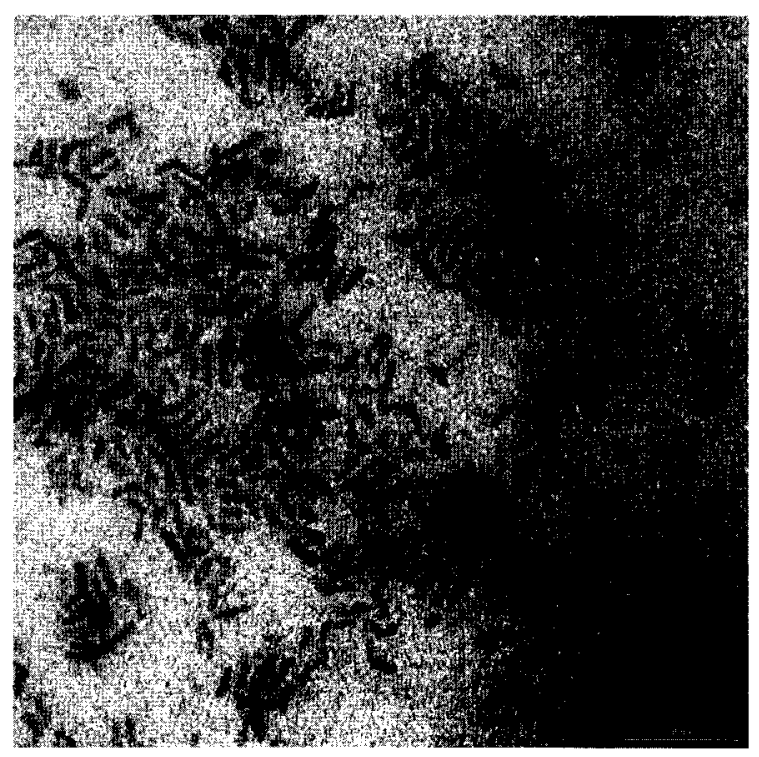

Fig. 1. TEM micrograph of CdSe nanorods grown at $240{ }^{\circ} \mathrm{C}$ for $5 \mathrm{~min}$. the width-depending on the growth temperature and time- was between 1 and $5 \mathrm{~nm}$. The FHWM of these particles was $35 \mathrm{~nm}$.

The different steps in the preparation of nanocrystaltubulin conjugates are displayed schematically in Figure 2. The $\mathrm{ZnS}$ (wurtzite) shell saturates dangling bonds and protects the CdSe core against oxidation, and the silica shell provides hydroxide groups for coupling of phosphonates and amines (water solubility). Finally, the tubulin is coupled to the nanoparticles.

Figure 3 depicts the absorption and photoluminescence spectra of the CdSe nanorods used for the labeling experiments. The particles were dispersed in chloroform (I), with wurtzite shell (II) and with silica shell in PBS (III). The emission maxima were found at $565 \mathrm{~nm}$ (I), $587 \mathrm{~nm}$ (II), and $590 \mathrm{~nm}$ (III). It was observed that the wurtzite passivation caused a redshift of the emission maximum of approximately $20 \mathrm{~nm}$. Furthermore, the photoluminescence quantum yield was increased. The redshift can be attributed to the electronic change of the particle surfaces; the increased luminescence, to the saturation of surface traps and dangling bonds. Compared with the wurtzite shell, the silica shell has small influence on the optical properties of the particles. These findings are in good agreement with the fact that the optical properties are mainly caused by the confinement of charge carriers within the nanocrystal core. Figure 3 also shows that the nanorods absorb light broadly in the UV up to approximately $550 \mathrm{~nm}$. From the onset value of the absorption, the effective band gap was estimated to be $2.17 \mathrm{eV}$.

These nanorods meet all the requirements for efficient labels for proteins such as tubulin. They are endowed with functional surface groups for the covalent coupling to proteins and they are soluble in water or biological buffers such as PBS. Furthermore, they show a broad absorption characteristic in the UV and a narrow, variable emission peak in the visible range of the spectrum. Therefore they satisfy the requirements for fluorescent labels suited for biological molecules.

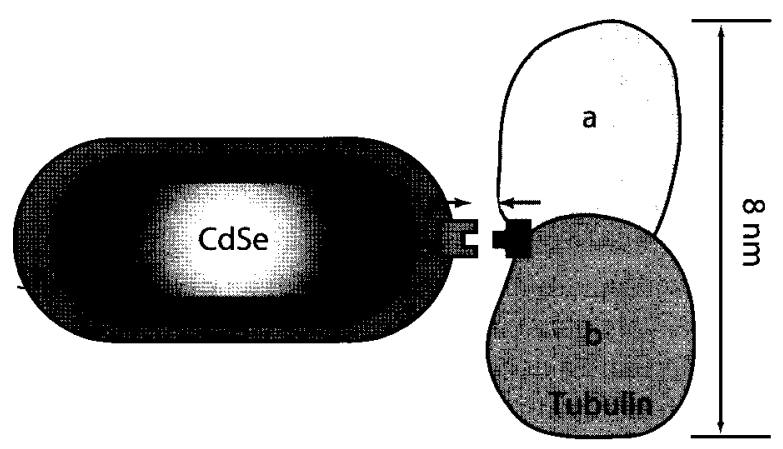

Fig. 2. Schematic description of the preparation process of nanorodcoupled neurotubulin. Grey area: CdSe nanorod; red: $\mathrm{ZnS}$ passivation shell; blue: silica shell with amine as tubulin-coupling group (green). The corresponding emission spectra are depicted in Figure 3. Nanoparticles are coupled covalently to tubulin by means of a peptide bond (green-red). 


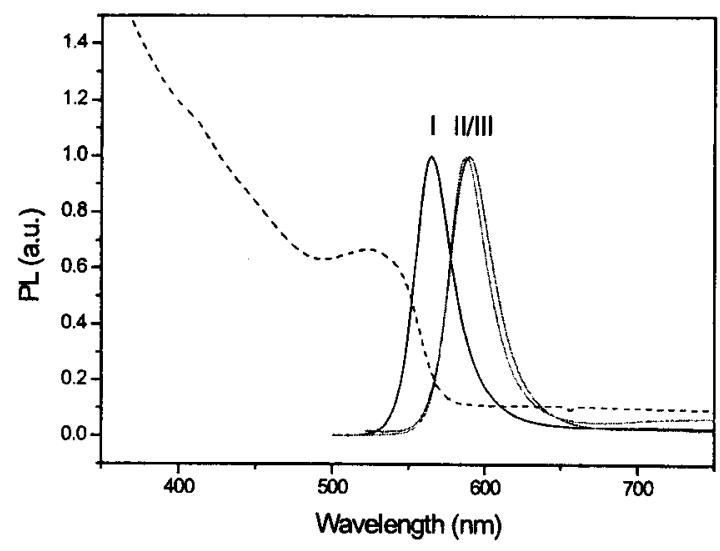

Fig. 3. Absorption (dotted line) and photoluminescence spectra of CdSe nanorods in chloroform (I), $\mathrm{ZnS}$-shelled CdSe nanorods in chloroform (II), and silica-shelled nanorods in PBS (III).

\subsection{Coupling of Nanorods to Tubulin}

To couple fluorescent nanorods to MAP-free tubulin, a simple standard protocol using cyanamide (50\% aqueous solution, 28335, pH 6.7; Fluka-Switzerland) was used that presumably links free carboxy groups (frequent at the carboxy terminus of $\alpha$-tubulin) to the amino groups on the surface of the nanorods. Preliminary studies (data not shown) demonstrated that the amount of protein in the assay was limiting. Preliminary studies (data not shown) had demonstrated that the yield of the final product increased with the initial concentration of tubulin. We therefore concentrated the tubulin to $3.5 \mu \mathrm{g} / \mu \mathrm{l}$. To preserve tubulin in a native configuration, the relative concentration of the coupling agent was reduced. Moreover, coupling at low temperature and in the presence of protease inhibitor as well as of antioxidant was found to produce a higher yield of soluble, coupled tubulin. Because the coupling was not sitespecific, it cannot be excluded that the coupled tubulin, although being still native, might have lost its ability to assemble into microtubules. This could be caused, for instance, when essential domains of tubulin (GTPase function, dimerization site) would be masked by the nanorods. To ensure that the coupled tubulin was still functional, it was driven through one cycle of warm-induced assembly and cold-induced disassembly. The fact that the supernatant from the warm-induced assembly was colorless, whereas the sediment was intensely fluorescent, indicates that most (if not all) of the coupled, soluble tubulin had remained functional. The final concentration of this potentially functional tubulin was around $1-1.5 \mu \mathrm{g} / \mu \mathrm{l}$ (i.e., around $30 \%$ of the initial tubulin could be recovered up to this stage). If other proteins are used where such a conditional assembly/disassembly protocol is not available, one might use a gel filtration column to separate the functional, soluble proteins from those molecules that have been degraded by the coupling process.

\subsection{Comparison: Nanorod and Rhodamine Fluorescence}

This was supported by the results from the assembly assay. Figure 3 shows that nucleation of microtubules became detectable from 2 min after assembly had been triggered by the addition of GTP. To prevent precocious assembly prior to the addition of GTP that would hamper the observation of the time course, MAP-free tubulin had to be used. In preliminary experiments, nanorod-labeled soluble tubulin was used at a concentration that was below the criticial threshold necessary for spontaneous microtubule assembly $(0.5 \mu \mathrm{g} / \mu \mathrm{l})$. In those experiments, the formation of fluorescently labeled microtubules could be triggered by the addition of $20 \mu \mathrm{M}$ taxol, a specific inhibitor of microtubule disassembly. Elongated filamentous microtubules coexisted with almost globular microtubule rods, irrespective of whether rhodamine or nanorods were used for visualization (Fig. 4A). The elongation of microtubules from these nucleation sites was proceeding rapidly in the time interval between 3 to $6 \mathrm{~min}$, when a kind of saturation was reached, which was probably due to depletion of tubulin heterodimers below the critical concentration needed for spontaneous assembly. The microtubule signal remained bright throughout this period and persisted even during the following hour under continuous irradiation (data not shown). In a parallel experiment, the assembly of rhodamine-coupled tubulin was followed as a reference. Again, nucleation was detected from 2-2.5 min and was followed by a phase of rapid microtubule elongation. However, from 4 min after the onset of the experiment, bleaching of rhodamine progressively impaired the visualization of microtubules, such that only the brightest regions of the microtubules remained visible. Because of this bleaching, the fine and rich network that was expected during the most active period of microtubule elongation could not be visualized properly. In other words, the use of fluorescent nanorods made it possible to follow the key events of microtubule assembly at high time resolution. In contrast, the bleaching of the conventional fluorophore affected the observation to an extent that the interesting time period, when microtubules elongate rapidly, was not accessible to continuous observation. The fact that the nanorod-coupled microtubules remained visible through at least $1 \mathrm{~h}$ of continuous irradiation suggests that it should be possible to follow assembly at a time resolution that would allow real-time imaging of the process.

\section{CONCLUSIONS}

The present study shows that the self-assembly of tubulin can be visualized by means of luminescent nanorods. (The use of rod-shaped nanocrystals could permit future experiments using circular dichroism.) Thus, for the first time, a dynamic subcellular process is visualized by nanorods. Photoluminescent nanorods were prepared with an organo- 
Rhodamine

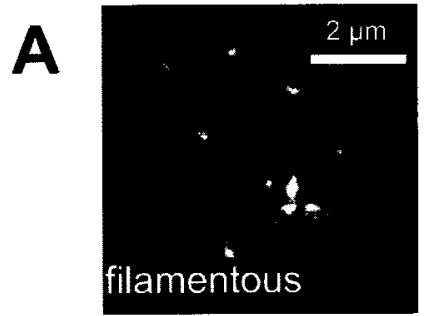

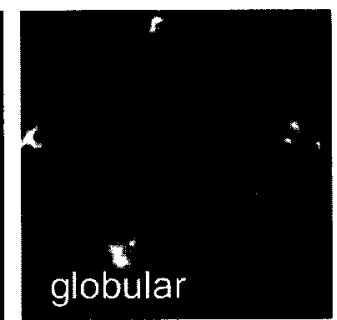

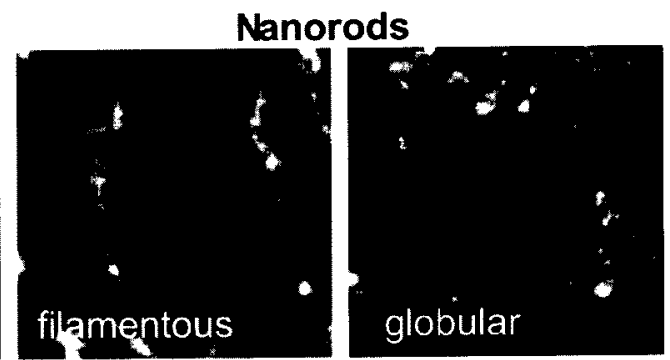

Time after addition of GTP [min]
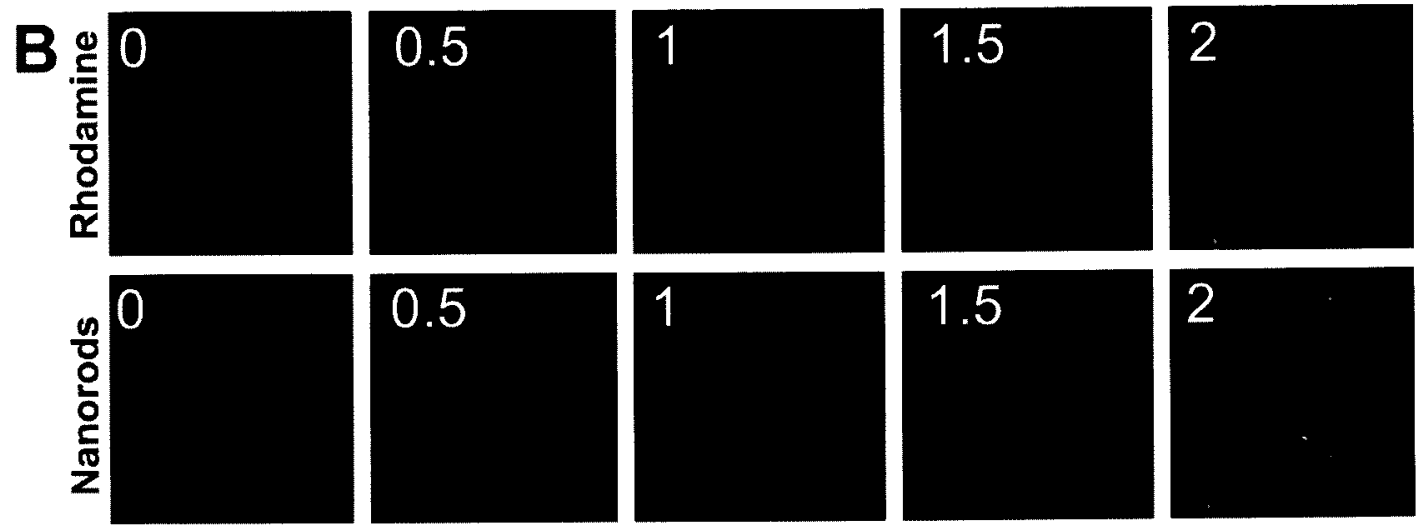

Time after addition of GTP [min]
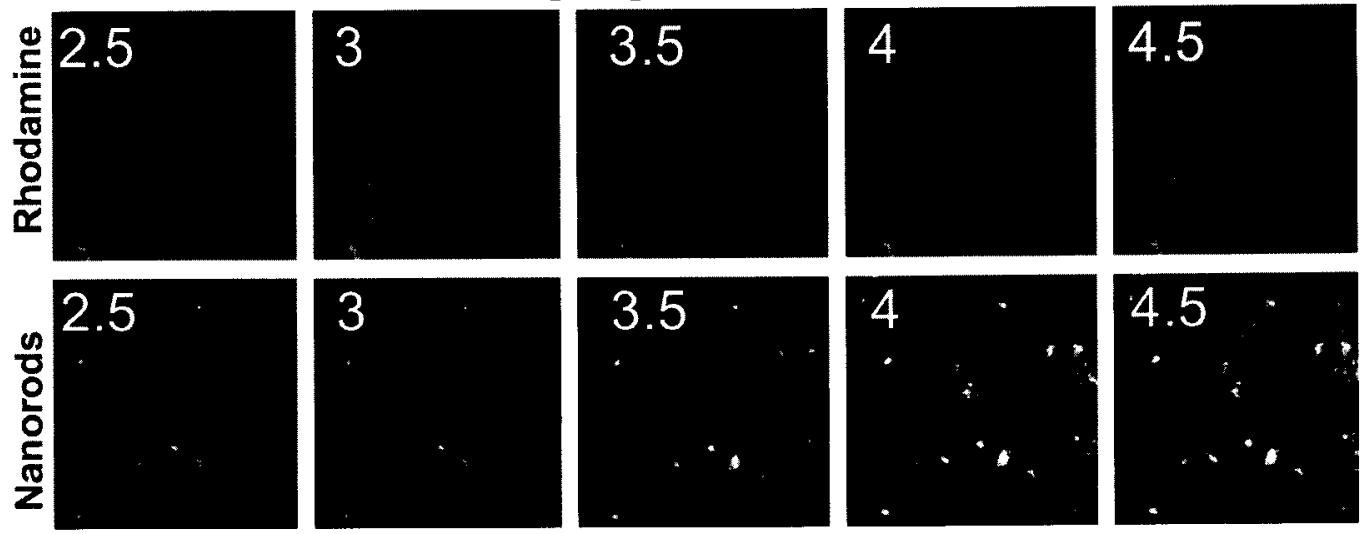

3
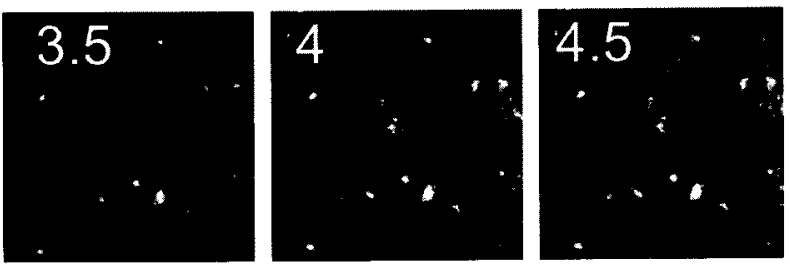

\section{Time after addition of GTP [min]}
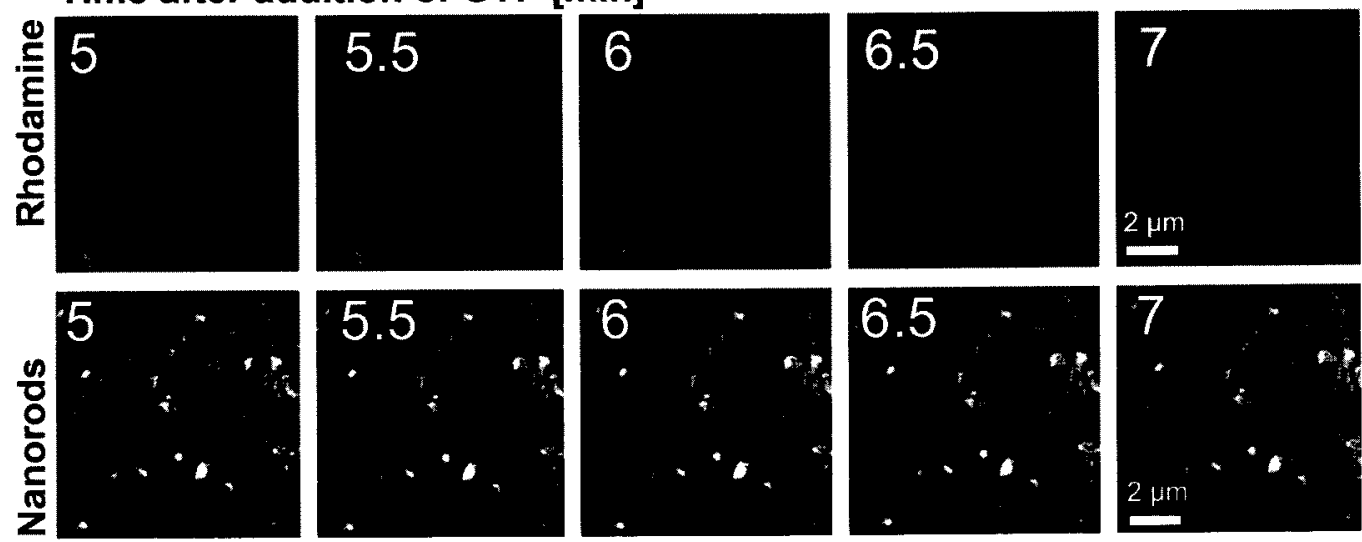

Fig. 4. Assembly of microtubules from fluorescently labeled neurotubulin in vitro. Microtubule assembly was triggered by the addition of GTP to the assay and followed over time. (A) Filamentous and more globular structures that coexist for both types of fluorophores. (B) Time course of assembly followed under continuous irradiation $\left(32 \mu \mathrm{mol} \cdot \mathrm{m}^{-2} \cdot \mathrm{s}^{-1}\right.$ ). Upper rows: neurotubulin that had been coupled to rhodamine as conventional fluorophore; lower rows: nanorod-coupled neurotubulin. 
metallic synthesis method and derivatized in a multilevel process such that the surface of the nanoparticles contained coupling groups for tubulin and surface charges to enable the solubilization in aqueous media. These nanocrystals were coupled covalently to tubulin and the conjugate was purified, making use of the biological function of the protein. The selfassembly of nanorod-labeled microtubules was induced and directly compared with rhodamine-labeled microtubules. The self-assembly of tubulin was found to be unaffected by the coupling to the nanorods. In addition, the use of nanorods made it possible to follow the dynamics of microtubule assembly under continuous irradiation, which cannot be achieved by conventional fluorophores, because of substantial bleaching. If one wanted to follow tubulin assembly at a similar higher time resolution with the use of conventional fluorophores, one would need to use intermittent frame acquisition, where short periods of illumination and data recording would be interrupted by intervening dark intervals to avoid bleaching. Although this is possible in conventional epifluorescence microscopy, this option is not available when confocal scanning has to be applied at high spatial resolution to visualize the fine structures of a given microtubule. Here, the bleaching becomes limiting for the temporal resolution that is possible. A substantial payoff of nanorod-coupled proteins as markers is therefore expected under conditions of high-resolution confocal imaging, where continuous excitation is needed to obtain a good time resolution. For instance, microinjection of nanorod-labeled tubulin into individual cells within their tissue context followed by appropriate biological stimulation ${ }^{19}$ should make it possible to follow microtubule responses at high spatiotemporal solution over the long time scales required to observe developmental events.

Acknowledgments: The authors thank Dr. Ralf Thomann for help with the TEM micrographs.

\section{References and Notes}

1. W. C. W. Chan and S. Nie, Science 281, 2016 (1998).

2. Z. Chunyang, M. Hui, N. Shuming, D. Yao, J. Lei, and C. Dieyan, Analyst 125, 1029 (2000).

3. H. Mattoussi. J. M. Mauro, E. R. Goldman, G. P. Anderson, V. C. Sundar, F. V. Mikulec, and M. G. Bawendi, J. Am. Chem. Soc. 122, 12142 (2000).

4. N. N. Mamedova, N. A. Kotov, A. L. Rogach, and J. Studer, NanoLetters 1, 281 (2001).

5. E. R. Goldman, E. D. Balighian, H. Mattoussi, M. K. Kuno, J. M. Mauro, P. T. Tran, and G. P. Andson, J. Am. Chem. Soc. 124, 6378 (2002).

6. S. Wang, N. Mamedova, N. A. Kotov, W. Chen, and J. Studer, NanoLetters 2, 817 (2002).

7. M. E. Åkerman, W. C. W. Chan, P. Laakkonen, S. N. Bhatia, and E. Ruoslahti, Proc. Natl. Acad. Sci. USA 99, 12617 (2002).

8. M. Bruchez, M. M. Moronne, P. Gin, S. Weiss, and A. P. Alivisatos, Science 281, 2013 (1998).

9. S. J. Rosenthal, I. Tomlinson, E. M. Adkins, S. Schroeter, S. Adams, L. Swafford, J. McBride, Y. Wang, L. J. DeFelice, and R. D. Blakely, J. Am. Chem. Soc, 124, 4586 (2002).

10. G. P. Mitchell, C. A. Mirkin, and R. L. Letsinger, J. Am. Chem. Soc. 121,8122 (1999).

11. S. Pathak, S.-K. Choi, N. Arnheim, and M. E. Thompson, J. Am. Chem. Soc. 123, 4103 (2001)

12. I. Willner, F. Patolsky, and J. Wasserman, Angew. Chem. 40, 1861 (2001).

13. D. Gerion, W. J. Parak, S. C. Williams, D. Zanchet, C. M. Micheel, and A. P. Alivisatos, J. Am. Chem. Soc. 124, 7070 (2002).

14. A. Schroedter, H. Weller, R. Eritja, W. E. Ford, and J. M. Wessels, NanoLetters 2, 1363 (2002).

15. W. J. Parak, D. Gerion, D. Zanchet, A. S. Woerz, T. Pellegrino, C. Micheel, S. C. Williams, M. Seitz, R. E. Bruehl, Z. Bryant, C. Bustamante, C. R. Bertozzi, and A. P. Alivisatos, Chem. Mater. 14,2113 (2002).

16. T. Nann and J. Riegler, Chem. Eur. J. 8, 4791 (2002).

17. A. Schroedter and H. Weller, Angew. Chem. 114, 3346 (2002).

18. M. L. Shelanski, F. Gaskin, and C. R. Cantor, Proc. Natl. Acad. Sci USA 70, 765 (1973).

19. R. Himmelspach, C. L. Wymer, C. W. Lloyd, and P. Nick, Plant J. 18,449 (1999).

Received: 13 February 2003. Revised/Accepted: 15 April 2003. 